\title{
O USO DOS FITOTERÁPICOS NO CONTROLE DO CÂNCER BUCAL: REVISÃO DE LITERATURA
}

\section{ARTIGO DE REVISÃO}

SILVA, Sheila Tayany Oliveira $\mathrm{Da}^{1}$

MONTENEGRO, Ziandra Soares ${ }^{2}$

SILVA, Sheila Tayany Oliveira Da. MONTENEGRO, Ziandra Soares. O uso dos fitoterápicos no controle do câncer bucal: Revisão de literatura, Revista Científica Multidisciplinar Núcleo do Conhecimento. Ano 05, Ed. 06, Vol. 13, pp. 54-66. Junho de 2020. ISSN: 2448-0959, Link de acesso: https://www.nucleodoconhecimento.com.br/saude/cancer-bucal

\section{RESUMO}

O presente estudo tem como base o índice de casos de câncer oral, que é o sexto tipo mais comum, sendo que em nível mundial é responsável por aproximadamente trezentos e noventa mil novos casos a cada ano, um dos mais preocupantes problemas de saúde pública, sendo considerado no contexto brasileiro como um dos dez mais corriqueiros em todo o país podendo ser identificado a partir da realização de um exame. Existem diversos fatores de riscos que podem se dar a partir de circunstâncias diversas, tais como os hábitos cotidianos, pois exite a premissa de que o câncer oral acompanha padrões de consumo tanto de tabaco quanto álcool que parecem estar associados à maioria dos casos apontados. É crescente o interesse de pesquisas com fitoterápicos, objetivando a produção de fármacos mais baratos e com efeitos semelhantes aos medicamentos tradicionais hoje existentes no mercado, dessa forma, estudos têm demonstrado que a medicina natural tem se apresentado como alternativa para propor novas modalidades terapêuticas com a utilização de

\footnotetext{
${ }^{1}$ Graduada em Odontologia pela Universidade Potiguar- UNP.

${ }^{2}$ Graduada em Odontologia pela Universidade Potiguar- UNP.
} 
fitoterápicos no tratamento de neoplasias. Tem como objetivo elucidar os possíveis mecanismos de ação dos fitoterápicos em relação à carcinogênese bucal, considerando-se os efeitos citotóxicos, pois serão fundamentais ao avanço das pesquisas voltadas aos fitoterápicos. A metodologia utilizada neste estudo baseou-se em uma estratégia qualitativa de pesquisa, de caráter exploratório, por meio de levantamento bibliográfico através de artigos de autores conhecidos e renomados. É possivel concluir que os baixos índices de sobrevida encontrados na literatura demonstram a necessidade de uma maior atenção à essas doenças, e é de grande importância que o cirurgião-dentista realize o reconhecimento das lesões cancerígenas para que o diagnóstico precoce seja eficaz. Essa revisão de literatura evidenciou que a os fitoterápicos podem ser mais uma alternativa para o tratamento e/ou a redução dos sintomas do câncer bucal, pois atuam como agentes quimiopreventivos, principalmente em relação à carcinogênese bucal, nota-se que é de extrema importância propor novas modalidades terapêuticas para o tratamento de neoplasias.

Palavras-chave: Neoplasia, bucal, fitoterápicos, evidências científicas, anticancerígeno.

\section{INTRODUÇÃO}

O número de casos de câncer tem aumentado mundialmente de maneira considerável, representando, na atualidade, um dos mais preocupantes problemas de saúde pública. O câncer oral, no contexto brasileiro, foi classificado como um dos dez mais comuns. A taxa de mortalidade encontra-se entre as mais elevadas do mundo. Desse modo, tal problema pode ser amenizado, pois, hoje, é possível detectar a presença desse tipo de câncer a partir de um exame rotineiro da cavidade oral. Em relação aos potenciais fatores de risco para o câncer bucal, o tabagismo e o etilismo parecem estar associados à maioria dos casos apontados. De acordo com literatura, podemos observar que, na maioria dos estudos, essa doença acomete indivíduos após a quinta década de vida, preferencialmente de raça branca e gênero masculino. Em 2013, foram registrados 5.401 óbitos em razão do câncer bucal, sendo 4.223 e 1178 do sexo masculino e feminino, respectivamente. 
De acordo com o Instituto Nacional do Câncer (INCA), a estimativa de novos casos chega a cerca de 15.490, sendo 11.140 homens e 4.350 mulheres para o ano de 2016 . As principais causas das neoplasias bucais são atribuíveis às influências ambientais, particularmente aquelas relacionadas ao estilo de vida, podendo ser induzidas por uma combinação de fatores: hábitos rotineiros; em razão do exercício do labor e em detrimento do local em que se habita. Nessa perspectiva, cabe destacar que a incidência desse tipo de câncer acompanha, também, padrões de consumo tanto de tabaco quanto de álcool historicamente. Além desses fatores, exposição às dietas pobres em frutas e vegetais, aos agentes biológicos, à irritação mecânica crônica, às radiações e à má higiene oral acarreta certas alterações de cunho epitelial que tornam a mucosa oral mais vulnerável aos agentes carcinógenos (SANTOS et al, 2010; WAAL, 2009).

Cabe auferir, também, que os tumores danosos à cavidade bucal, em sua maioria, são constituídos a partir do que se conhece como carcinoma epidermoide e este é classificado em alguns níveis, sendo eles: pouco, moderado e bem diferenciado. $O$ local de maior acometimento pelo carcinoma intra oral é a língua, e, geralmente, é comum a sua manifestação nas superfícies lateral posterior e ventral. O soalho bucal é acometido mais frequentemente nos homens. Em ordem decrescente de frequência podemos citar o palato mole, gengiva, mucosa jugal e palato duro. Todavia, mesmo que apresentem uma incidência menos significativa, é pertinente apontar, aqui, outros tipo de tumores comuns à cavidade oral. São eles os tumores salivares(das glândulas salivares menores e da sublingual), os sarcomas (os de origem vascular, os musculares e os ósseos) e o melanoma de mucosa, conforme indicada o estudo de Neville et al (2009).

As pesquisas recentes apontam, ainda, no que tange a direção de fármacos que interferem na inibição do desenvolvimento tumoral, que estes podem atuar visando o aumento significativo da sobrevida de pacientes portadores de neoplasias malignas. Contudo, tais fármacos podem, ainda, exercer ação sobre a viabilidade das células tumorais e, também, no combate imunológico as doenças (AVELAR, 2010; CAVALCANTE, 2011; KANG et al, 2009; PEDREIRA, 2007). Nesse sentido, pode-se 
auferir que é crescente o interesse de pesquisas com fitoterápicos, sobretudo aquelas que objetivam a produção de fármacos mais baratos e com efeitos semelhantes aos medicamentos tradicionais hoje existentes no mercado. Dessa forma, estudos têm demonstrado que a medicina natural tem se apresentado como alternativa para propor novas modalidades terapêuticas com a utilização de fitoterápicos no tratamento de neoplasias.

Apesar do uso tradicional e frequente por parte da população a real efetividade, os princípios ativos e a ausência de toxicidade ainda não estão totalmente esclarecidos. No entanto, o interesse pelos extratos de plantas e vegetais utilizados na medicina popular tem crescido, tendo-se, como objetivo, descobrir novos compostos com diferentes efeitos farmacológicos, e menores efeitos colaterais quando comparados aos fármacos já existentes (MANOHARAN et al, 2006; MENDONÇA et al, 2015; OLIVEIRA; EVANGELISTA-COIMBRA, 2014; PEDREIRA, 2007). Os estudos farmacológicos dos fitoterápicos comprovam a sua eficácia e toxicidade com o objetivo de traçar o perfil dos efeitos colaterais, definindo um mecanismo seguro de ação, relacionando os seus efeitos às doses para que possam ser utilizados de forma segura em humanos (AVELAR, 2010; CAVALCANTE, 2011; KAVITHA et al, 2006; RENJU et al, 2007).

Novos estudos com o intuito de elucidar os possíveis mecanismos de ação dos fitoterápicos em relação à carcinogênese bucal, considerando-se os efeitos citotóxicos, serão fundamentais ao avanço das pesquisas voltadas aos fitoterápicos. Baseado nos inúmeros estudos que apresentam evidencias científicas na atividade anticarcinogênica, julgou-se válido realizar uma revisão de literatura para elucidar sobre o tema abordado.

\section{REFERENCIAL METODOLÓGICO}

Para a realização deste trabalho, inicialmente, foram escolhidos fitoterápicos utilizados no tratamento de doenças humanas. Essa escolha se deu a partir da base de dados de um site de pesquisa, onde pesquisamos o uso de plantas no tratamento de câncer. Após esses dados estarem disponíveis. Selecionou-se uma pesquisa 
específica sobre os fitoterápicos escolhidos. Foram investigadas informações sobre o nome científico e os tipos de câncer que já foram tratados. Outra revisão bibliográfica foi realizada a partir do acervo virtual do Sistema Integrado de Bibliotecas - SIB e contemplou-se as bases de dados da Medline, Sience Direct, UpToDate, BVS, Scielo e muitas outras. Os nomes científicos e os nomes de conhecimento popular foram utilizados para efetivação da pesquisa nas bases de dados.

Durante a pesquisa, as bases de dados escolhidas retornaram uma diversidade de artigos relacionados ao nome científico, no entanto, apenas 09 artigos estavam relacionados ao estudo do tratamento do câncer, e, desse modo, essa quantidade que, em um primeiro momento, era maior, foi reduzida aos 09 artigos relacionados ao escopo do estudo. Para que se obtivessem artigos satisfatórios para o foco do trabalho adicionou-se palavras-chave ideais à este estudo (câncer bucal, ratos e a denominação científica de cada fitoterápico). Na seleção da literatura a ser utilizada foram contemplados os artigos que estavam disponíveis com o acesso completo e que apresentavam relevância ao assunto pesquisado, ou seja, relacionados ao uso de fitoterápicos que apresentavam ação anticarcinogênica no câncer bucal em ratos.

\section{ASPECTOS GERAIS SOBRE O CÂNCER}

No corpo humano há um mecanismo responsável pelo controle do crescimento de diferentes células para que consigam atingir um tamanho específico e uma proliferação ordenada. Podemos encontrar um mecanismo de reparo e suporte, que, por sua vez, faz com que seja possível a recuperação do tecido danificado decorrente de alguma lesão. De acordo com Otto (2002), o câncer pode ser definido como um processo lógico em que as células normais sofrem modificações e passam a ter capacidade especiais. Essas células, quando modificadas, são denominadas de células cancerígenas e a proliferação desordenada de células defeituosas presentes em nosso organismo irá determinar um novo comportamento e um crescimento mais acelerado delas.

Conforme os tecidos vão sendo invadidos, eles perdem as suas funções, desenvolvendo, no indivíduo, sintomas característicos de acordo com o local atingido. 
O resultado desse acúmulo de células defeituosas é a formação de tumores, que, por sua vez, podem ser classificados como benignos ou malignos. Atualmente, um dos principais desafios para os países em desenvolvimento é definir e implementar estratégias efetivas para o controle do câncer. No Brasil, o câncer representa mais da metade do total dos óbitos e o número de indivíduos acometidos pelo câncer bucal tem aumentado gradativamente (MATEUS, 2008). O câncer oral no contexto brasileiro é um dos dez mais frequentes na população. A taxa de mortalidade encontra-se entre as mais elevadas do mundo. No ano de 2013 houve 5.401 óbitos, sendo 4.223 homens e 1.178 mulheres.

Nesse sentido, cabe destacar que, atualmente, é possível detectar, de modo precoce, a presença ou ausência do câncer bucal, bastando realizar um exame rotineiro da cavidade bucal. Em relação aos potenciais fatores de risco para o câncer bucal, o tabagismo e o etilismo parecem estar associados à maioria dos casos apontados. De acordo com literatura, é possível auferir, com base nas observações, que a maioria dos estudos selecionados apontam que essa doença acomete indivíduos após a quinta década de vida, preferencialmente de raça branca e gênero masculino (INCA, 2016). Para melhorar o prognóstico e qualidade de vida dos pacientes, os profissionais de saúde devem estar atentos aos sinais e sintomas da doença, para que, dessa forma, o diagnóstico possa ser o mais rápido e preciso possível, agilizando, assim, o início do tratamento contra o câncer.

\subsection{O USO DE PLANTAS MEDICINAIS E AS SUAS PRINCIPAIS CARACTERÍSTICAS}

Atualmente, uma grande parcela da população que é portadora de câncer recorre à possibilidades múltiplas na busca pela cura, e, muitas dessas pessoas, têm sido cada vez mais adeptas à medicina natural. Entretanto, Elias, Alves e Tubino (2006) apontam para o fato de que apesar desse uso ter se expandido, o funcionamento desse vértice da Medicina ainda tem sido estudado e ele não é conhecido pela maioria das pessoas, pois há pouca divulgação desse possibilidade. Contudo, há que se destacar que o uso de plantas medicinas não é algo recente, pois são as formas mais 
antigas empregadas pelo homem no tratamento de doenças dos mais diversos tipos. Sendo assim, o uso de plantas como forma de prevenir doenças e a cura delas é uma prática que já existe há bastante tempo na história da humanidade (MORAES; SANTANA, 2001 apud FIRMO et al, 2011). Atualmente, é crescente o interesse de pesquisas com fitoterápicos, objetivando a produção de fármacos mais baratos e com efeitos semelhantes aos medicamentos tradicionais existentes no mercado.

Dessa forma, estudos apontam que a medicina natural é uma alternativa para propor novas modalidades terapêuticas com a utilização de fitoterápicos no tratamento de neoplasias. Apesar do uso tradicional e frequente por parte da população, a real efetividade, os princípios ativos e a ausência de toxicidade ainda não estão totalmente esclarecidos. No entanto, o interesse pelos extratos de plantas e vegetais que pertencem à esfera da medicina popular tem crescido, tendo-se, como objetivo, descobrir novos compostos com diferentes efeitos farmacológicos e menores efeitos colaterais quando comparados aos fármacos já existentes (MANOHARAN et al, 2006; MENDONÇA et al, 2015; OLIVEIRA; EVANGELISTA-COIMBRA, 2014; PEDREIRA, 2007). Os estudos farmacológicos dos fitoterápicos comprovam a sua eficácia e toxicidade com o objetivo de traçar o perfil dos efeitos colaterais, definindo um mecanismo seguro de ação, relacionando os seus efeitos às doses para que possam ser utilizados de forma segura em humanos (AVELAR, 2010; CAVALCANTE, 2011; KAVITHA et al, 2006; REJUN et al, 2007).

Nas últimas décadas, a utilização dos fitoterápicos elevou-se consideravelmente em todo o mundo, e, portanto, a sua discussão nessa reflexão tem a sua relevância comprovada. Segundo dados da Organização Mundial de Saúde (OMS), pode-se afirmar que, aproximadamente, $80 \%$ da população mundial utiliza algum tipo de erva. As ervas são utilizadas quando se deseja buscar o alívio de algum sintoma doloroso. Do total dos dados apresentados pela OMS, cerca de 30\% do uso de ervas se deu em razão de alguma indicação médica. Nesse sentido, cabe auferir que o uso de plantas medicinas tem sido incentivado, inclusive, pela própria OMS. Apesar de se ter um grande número de pesquisas relacionadas à este tipo de medicamento e a maior reserva de biodiversidade do mundo, o Brasil ainda não possui um mercado 
expressivo em relação aos fitoterápicos, pois, em alguns casos, esse mercado é menos expressivo do que os daqueles com menor desenvolvimento tecnológico. Incentivar essa cultura é uma das metas.

\subsection{ASPECTOS POSITIVOS PARA UTILIZAÇÃO DOS FITOTERÁPICOS}

A grande variedade de diferentes espécies vegetais nativas e o fácil acesso que os usuários podem ter é um dos fatores que levam as pessoas a mostrarem preferência pela medicina alternativa. Pode-se citar, também, o menor custo no preparo de infusões e chás, visto que proporciona vantagens para as populações de baixa renda, podendo o usuário fazer o cultivo dessas plantas (MORAES; ALONSO; OLIVEIRAFILHO, 2011). Os fitoterápicos, em relação à medicina moderna são mais úteis quando utilizados em determinados tratamentos de enfermidades crônicas, pois há menos efeitos colaterais e são mais baratos. Outro benefício é que os fitoterápicos podem ser associados aos medicamentos alopáticos. No entanto, é necessário cuidado em relação a associação, pois os medicamentos fitoterápicos podem potencializar os efeitos de alguns medicamentos convencionais. Esse uso deve ser feito sempre com o devido acompanhamento (ARNOUS; SANTOS; BEINNER, 2005).

\subsection{ASPECTOS NEGATIVOS PARA UTILIZAÇÃO DOS FITOTERÁPICOS}

Os princípios ativos de plantas medicinais são responsáveis pelas suas propriedades terapêuticas como, também, pelas suas intoxicações e reações adversas que podem apresentar-se devido ao uso em doses desnecessárias ou por períodos prolongados. Pelo fato da ausência de esclarecimentos objetivos e atualizados correspondentes aos possíveis riscos e benefícios do uso de plantas medicinais, deve ser considerado um dos principais fatores contribuintes para a população fazer uso da automedicação. Por serem medicamentos naturais, a grande das maiorias pessoas pensam que a utilização das plantas medicinais é uma forma mais segura e acabam utilizando-as de forma incorreta (BASTOS, 2007). 
Supõe-se que o uso de plantas medicinais é uma ação inofensiva à saúde, contudo, a pesquisa aqui proposta enfatiza que a sua má administração pode acarretar resultados danosos, levando em conta que uma mesma planta pode apresentar uma ação tanto terapêutica quanto tóxica, definida pela dosagem utilizada e pela forma em que é preparada (GOMES et al, 2001). De acordo com Turolla e Nascimento (2006), várias plantas apresentam substâncias que podem apresentar efeitos colaterais em seu uso de forma errada. Tais substâncias, em sua maioria, são associadas à componentes ou à contaminantes e adulterantes que estão presentes nos preparos fitoterápicos, o que exige uma minuciosa coleta, uma boa qualidade do cultivo das plantas, cuidado com a extração de componentes e com o produto final.

\section{DISCUSSÃO}

\subsection{O USO DE FITOTERÁPICOS NO TRATAMENTO DE VÁRIOS TIPOS DE CÂNCER}

Muitos estudos multidisciplinares envolvendo diversas áreas profissionais como etnobotânicos, químicos, farmacêuticos, patologistas, homeopatas foram desenvolvidos nos últimos anos, sendo conduzidos no sentido de ampliar o conhecimento sobre as plantas medicinais, com a finalidade de avaliar as interações com medicamentos e diversos tratamentos já utilizados no tratamento contra o câncer, bem como os possíveis efeitos citotóxicos e sua ação anticarcinogênica. Em seu estudo de indução ao câncer de pele em camundongos sírios albinos Das et al (2008) demonstraram que a ação do açafrão pode inibir a proliferação celular, bem como hiperplasia, displasia e o crescimento do papiloma. Observou-se, também, que a formação de microvasos foi inibida em papilomatose pela ingestão de açafrão, o que sugere que o açafrão pode prevenir ou retardar a angiogênese e a progressão de tumores.

Um certo número de estudos tanto in vitro como in vivo demonstraram a capacidade de romã e seus polifenóis para contrastar vários eventos biológicos envolvidos na patogênese e progressão do cancro. Este cenário abre o caminho para uma dupla 
exploração dos polifenóis da romã no cancro: como uma estratégia quimiopreventivo para reduzir o aparecimento de tumores por meio de agentes dieta e anticancerígenos direta para tratar diferentes tumores humanos em regimes de dosagem mais elevados de forma mais confiável, alcançáveis a partir da entrega farmacêutica de compostos purificados (TURRINI et al, 2015). A administração oral da Clerodendron inerme apresentou propriedades quimiopreventivas e funções antioxidantes das células de ratos albinos DMBA induzidas em um estudo feito por Renju et al (2007).

\subsection{O USO DE FITOTERÁPICOS E O CÂNCER BUCAL}

Nos últimos anos, tem-se observado que o interesse pelos extratos de plantas vegetais utilizados na medicina popular tem crescido, e, desse modo, tem-se como objetivo descobrir novos compostos com diferentes efeitos farmacológicos e menor efeito indesejável quando comparados aos fármacos já existentes. Apesar do uso tradicional, é frequente, por parte da população, duas questões precisam ser aprofundadas: real efetividade e ausência de toxicidade. É necessário conhecer a dose eficaz e aquela que produz toxicidade, sendo fundamental a instituição de um estudo farmacodinâmico e toxicológico padronizado (CAVALCANTE, 2011; PEDREIRA, 2007; KAVITHA et al, 2006; KANG et al, 2009; RENJU et al, 2007).

Embora o Brasil seja um país rico em Biodiversidade e domine a maioria das tecnologias para a produção de fitomedicamentos, há um elevado grau de desconhecimento da classe médica acerca dos princípios ativos, indicações e contraindicações desses fitoterápicos; somado a visão de descrença da eficácia de ação desses produtos nas diversas doenças que afetam a população (PEDREIRA, 2007). O esforço para combater o câncer levou a muitas descobertas fundamentais no campo da biologia celular, inclusive de várias proteínas que possuem anormalidades na sua função e que podem levar a um crescimento desordenado das células, aumentando a divisão, diminuição da morte celular ou outras características aberrantes das células cancerosas.

Essas proteínas podem estar envolvidas na reparação do DNA, na sinalização, ciclo e crescimento celular, bem como na morte programada das células (ALBERTS et al, 
2008). Pesquisas têm sido desenvolvidas a partir do estudo dos carotenos que atuam como agentes quimiopreventivos, principalmente em relação a carcinogênese; com maior ênfase aos betacarotenos que comprovadamente atuam prevenindo a iniciação e progressão de várias formas de cânceres humanos (MANJANATHA, 2006). Estudos realizados com a Tephrosia purpurea indicam, portanto, que este fitoterápico atua como um poderoso quimiopreventivo contra o carcinoma bucal ocasionado por DMBA. Além disso, a Tephrosia purpurea melhorou o estado de antioxidantes na circulação de DMBA em hamsters.

Foi observada, também, uma melhoria no sistema de defesa antioxidante na mucosa bucal de hamsters após o tratamento com Tephrosia purpurea (KAVITHA et al, 2006). Destarte, pesquisas diversas têm apontado a eficiência do uso de plantas medicinais no que tange o tratamento do câncer bucal (CHEN et al, 2014; DAS et al, 2010; KASEM et al, 2014). Muitos desses estudos demonstram a utilização combinada com fitoterápicos e outros tratamentos já utilizados, podendo ser discriminada a potencialização dos efeitos benéficos.

\section{3 ÓLEO RESINA DE COPAÍBA}

Avaliações "in vivo" e "in vitro" têm demonstrado que os óleos de várias espécies de copaíferas possuem atividade anti-inflamatória, cicatrizante, antiedematogênica, antitumoral e bactericida, sendo crescente seu uso como fitoterápico, comprovando, cientificamente $_{2}$ a eficácia do óleo resina de Copaíba. Veiga Jr e Pinto (2005) avaliaram a diminuição do edema da pata de ratos que foram tratados com o óleo e observaram, também, uma acentuada redução no mecanismo de permeabilidade vascular. A ideia é reforçada por Pedreira (2007), pois, o seu estudo, aufere que o óleo pode ter promovido alguma ação inibitória na formação do câncer bucal induzido por DMBA em 80 hamsters. Aspectos macroscópicos e microscópicos apontaram, ainda, uma significativa redução nos grupos, mostrando a importância do aprofundamento sobre essa substância. 


\subsection{A PRÓPOLIS}

A própolis é conhecida há séculos por suas propriedades de cicatrização, mas recentemente tem atraído a atenção de cientistas devido às suas atividades de amplo aspectro que podem ser usados na medicina complementar e alternativa. Consistentemente, o número de estudos realizados com esta substância exibiu um aumento significativo nos países sem o uso tradicional da medicina natural (MENDONÇA et al, 2015). Os resultados da pesquisa realizada por Cavalcante et al (2011) sugerem uma possível atividade quimiopreventiva e antitumoral da própolis verde. Outro estudo demonstrou que a própolis exibiu atividade antioxidante e em maior concentração nas linhas celulares tumorais humanas. Portanto, pode ser útil para o desenvolvimento de novos medicamentos e fitomedicamentos para o tratamento do câncer (MENDONÇA et al, 2015).

\subsection{AVELOZ}

Tendo em vista os altos níveis de compostos bioativos presentes nas plantas do gênero Euphorbia, é provável que algumas espécies possam realmente apresentar atividades biológicas capazes de promover uma melhora clínica em algumas situações patológicas (FERNANDEZ et al, 2010; LAGE et al, 2009).

\section{CONSIDERAÇÕES FINAIS}

Baseado neste estudo é lícito concluir que:

1. Os baixos índices de sobrevida encontrados na literatura demonstram a necessidade de uma maior atenção à essas doenças, bem como o uso de recursos alternativos, como a medicina não convencional;

2. É de grande importância que o cirurgião-dentista realize o reconhecimento das lesões cancerígenas para que o diagnóstico precoce seja eficaz;

3. O uso de fitoterápicos é uma prática muito antiga, e, atualmente, tem se destacado em razão do aumento significativo de usuários que buscam um estilo 
de vida saudável, tantos nas camadas sociais mais baixas da população quanto nas mais altas;

4. Considerando os benefícios apontados em diversas pesquisas realizadas nos últimos anos, essa revisão de literatura mostrou que a os fitoterápicos podem ser mais uma alternativa para o tratamento e/ou a redução dos sintomas do câncer bucal. Os resultados positivos ressaltam a necessidade de aprofundamento sobre o tema e apoio à novos estudos para que seja possível o avanço das pesquisas na área fitoterápica.

\section{REFERÊNCIAS}

ALBERTS, B. et. al. Molecular biology of the cell. $5^{\mathrm{a}}$ ed. New York: Garland Science, 2008.

ARNOUS, A. H.; SANTOS, A. S.; BEINNER, R. P. C. Plantas medicinais de uso caseiro: conhecimento popular e interesse por cultivo comunitário. Espaço para a Saúde, v. 6, n. 2, p. 1-6, jun. 2005.

AVELAR, B. A. de. Detecção in vitro de citocinas intracitoplasmáticas (interferon gama, fator de necrose tumoral, interleucina 4 e interleucina 10) em leucócitos humanos tratados com extrato bruto diluído de euphorbia tirucalli. 2010. $79 \mathrm{f}$. Dissertação (Mestrado em Ciências Fisiológicas) - Universidade Federal dos Vales do Jequitinhonha e Mucuri, Diamantina, 2010.

BASTOS, G. M. Uso de preparações caseiras de plantas medicinais utilizadas no tratamento de doenças infecciosas. 2007. 111 f. Dissertação (Mestrado em Ciências Farmacêuticas. Farmácia Clínica) - Faculdade de Farmácia, Ondotologia e Enfermagem, Universidade Federal do Ceará, Fortaleza, 2007.

CAVALCANTE, D. R. R. et al. Effect of green propolis on oral epithelial dysplasia in rats. Braz J Otorhinolaryngol. v.77, n.3, p. 278-84, 2011. 
CHEN, C. et al. Curcumin Suppresses Metastasis via Sp-1, FAK Inhibition, and ECadherin Upregulation in Colorectal Cancer. Evidence-based Complementary And Alternative Medicine, v. 2013, p.1-17, jun. 2013.

CHEN, Y-W. et al. Enhanced Anti-Tumor Activity of Triptolide inCombination with Irradiation for the Treatment of Oral Cancer. Georg Thieme Verlag Kg Stuttgart, v. 4, n. 80, p.255-261, jan. 2014.

CHORILLI, M; MICHELIN, D.C; SALGADO, H. R. N. Animais de laboratório: o camundongo. Revista de Ciências Farmacêuticas Básica e Aplicada, v. 28, n. 1, p.11-23, jul. 2007.

DAS, I. et al. Saffron suppresses oxidative stress in DMBA-induced skin carcinoma: A histopathological study. Acta Histochemica, v. 112, n. 4, p.317-327, jul. 2010.

ELIAS, M. C.; ALVES, E.; TUBINO, P. Uso de medicina não convencional em Crianças com câncer. Revista Brasileira de Cancerologia, v. 52, n.3, p. 237-243, 2006.

FERNANDEZ-ARCHE, A. et al. Topical anti-inflammatory effect of tirucallol, a triterpene isolated from Euphorbia lactea latex. Phytomedicine, v. 17, n. 2, p. 146148, fev. 2010.

FIRMO, W. C. A., et al. Contexto histórico, uso popular e concepção científica sobre plantas medicinais. Cad. Pesq., v. 18, n. especial, dez. 2011.

GOMES, E. C. et al. Plantas medicinais com características tóxicas usadas pela população do município de Morretes, PR. Visão Acadêmica, v. 2, n. 2, p.77-80, jul./dez. 2001.

INSTITUTO NACIONAL DO CÂNCER - INCA. Disponível em: http://www2.inca.gov.br/wps/wcm/connect/tiposdecancer/site/home/boca/definicao. Acesso em: 03 mar. 2016. 
KANG, J. W. et al. Kaempferol and quercetin, components of Ginkgo biloba extract (EGb 761), induce caspase-3-dependent apoptosis in oral cavity cancer cells. Phytother. Res., v. 24, n. 1, p.77-82, 7 jul. 2009.

KASEM, R. F. et al. Chemopreventive effect of Mentha piperita on dimethylbenz[a]anthracene and formaldehyde-induced tongue carcinogenesis in mice (histological and immunohistochemical study). J Oral Pathol Med, p.484-491. jan. 2014.

KAVITHA, K.; MANOHARAN, S. Anticarcinogenic and antilipidperoxidative effects of Tephrosia purpurea (Linn.) Pers. in 7, 12-dimethylbenz(a)anthracene (DMBA) induced hamster buccal pouch carcinoma. Indian Journal of Pharmacology, v. 38, n. 3, p.185-9, 2006.

LAGE, H. et al. Antitumor activity of terpenoids against classical and atypical multidrug resistant cancer cells. Phytomedicine, v. 17, n. 6, p.441-448, Maio, 2010.

MANJANATHA, M. G. Dietary effects of soy isoflavones daidzein and genistein on7, 12-dimethylbenz[a]anthracene-induced mammary mutagenesis and carcinogenesis in ovariectomized Big Blue transgenic rats. Carcinogenesis, v. 27, n. 12, p. 2555-2564, 2006.

MANOHARAN, S. et al. Clerodendron Inerme Protects Cellular Integrity during 7,12 Dimethylbenz[A]-Anthracene Induced Hamster Buccal Pouch Carcinogenesis. Afr J Tradit Complement Altern Med, v. 5, n. 2, p. 213-222, 2008.

MANOHARAN, S. et al. Evaluation of anticarcinogenic effects of Clerodendron inerme on 7, 12-dimethylbenz[a]anthracene-induced hamster buccal pouch carcinogenesis. Singapore Med J, v. 47, n. 12, p. 1038-1043, dez. 2006.

MATEUS, F. O. Câncer Bucal no Brasil: Revisão de Literatura. 2008. 29f. Monografia (Especializaçã em Medicina) - Universidade Federal do Rio Grande do Sul, Porto Alegre, 2008. 
MENDONÇA, I. C. G. De. et al. Brazilian red propolis: phytochemical screening, antioxidant activity and effect against cancer cells. BMC Complementary and Alternative Medicine, v.15, n. 357, 2015.

MORAES, L. G. de; ALONSO, A. M.; OLIVEIRA-FILHO, E. C. Plantas medicinais no tratamento do câncer: uma breve revisão de literatura. Universitas: Ciências da Saúde, v. 9, n. 1, p. 77-99, jan./jun. 2011.

NEVILLE, B. W. et al. Patologia Oral e Maxilofacial. $3^{a}$ ed. Rio de Janeiro: Elsevier, 2009.

OLIVEIRA, B. M. de; EVANGELISTA-COIMBRA, C. C. B. Euphorbia tirucalli: no tratamento complementar do Câncer. Revista UningÁ Review, v. 20, n. 3, p. 60-64, out. 2014.

OTTO, S. E. Oncologia. 2 ${ }^{\mathrm{a}}$ ed. Rio de Janeiro: Reichmann \& Affonso, 2002.

PEDREIRA, E.N. Avaliação do efeito inibidor tumoral do óleo resina de Copaíba in natura (copaifera reticulata) e manipulado artesanalmente no modelo de carcinogênese bucal experimental Dmba induzida. 2007.169f. Tese (Doutorado em Odontologia) - Faculdade de Odontologia de Bauru. Universidade de São Paulo, São Paulo, 2007.

RENJU, G. L. et. al. Chemopreventive and antilipidperoxidative potential of Clerodendron inerme (L) Gaertn in 7,12-dimethylbenz(a)anthracene induced skin carcinogenesis in Swiss albino mice Pak. J. Biol. Sci., v.10, p.1465-1470, 2007.

SANTOS, L. C. O. et. al. Characterization of oral cancer diagnostic delay in the state of Alagoas. Brazilian Journal Of Otorhinolaryngology, v. 76, n. 4, p. 416-422. jun. 2010.

TUROLLA, M. S. R.; NASCIMENTO, E. S. Informações toxicológicas de alguns fitoterápicos utilizados no Brasil. Revista Brasileira de Ciências Farmacêuticas, v. 42, n. 2, p. 289-306, abr./jun. 2006. 
TURRINI, E.; FERRUZZI, L.; FIMOGNARI, C. Potential Effects of Pomegranate Polyphenols in Cancer Prevention and Therapy. Oxidative Medicine And Cellular Longevity, [s.I.], v. 2015, p.1-19, jun. 2015.

VEIGA JÚNIOR, V. F.; PINTO, A. C. Plantas medicinais: cura segura? Química Nova, v. 28, p. 519-528, 2005.

WAAL, I. V. D. Potentially malignant disorders of the oral and oropharyngeal mucosa; terminology, classification, and present concepts of management. Oral Oncology, v. 45, n. 4-5, p. 317-323, abr. 2009.

Enviado: Maio, 2020.

Aprovado: Junho, 2020. 